\title{
Using Online Photo Sharing to Support Parents with Type One Diabetic Children
}

\section{Hajar Alamri}

Department of Computer Science and Information Systems

King Khalid University

Abha, Saudi Arabia

\&

Department of Computing and Information Systems

University of Melbourne

Victoria, Australia

Email: halamri@student.unimelb.edu.au

\section{Reeva Lederman}

Department of Computing and Information Systems

University of Melbourne

Victoria, Australia

Email: reeva.lederman@unimelb.edu.au

\section{Rachelle Bosua}

BPM and IT Group

Open University of The Netherlands

Netherlands

Email: rachelle.bosua@ou.nl

\section{Abstract}

The rise of online photo sharing platforms has encouraged an increasing number of people to share online their lived experience as a carer of a child with a chronic condition. This study aims to explore the effectiveness of using online photo sharing platforms to support parents of chronically ill children. We use individual interviews and an analysis of Instagram posts to study parents of Type 1 diabetic children who actively engage in photo sharing. Findings show online photo sharing supports parents in coping with their child's condition and in sharing their experience with and supporting other parents. Photos have a significant capacity to deliver personal experience and therefore in enhancing relationships between participants. Participants express the greater ability of photos over text in validating their experience. More specifically, personal photos create more trust in the provided information. This supports the idea that photo sharing can provide opportunities for emotions-focused coping skills, not as easily provided by text.

Keywords Coping theory, Photo sharing, Parent, Carer, Instagram, Type 1 Diabetic. 


\section{Introduction}

Online photo sharing through Instagram has grown significantly as a social media service on the Internet. Since its debut in 2010, more than 300 million Instagram stories have been shared by active users on a daily basis (Instagram 2018). Although Instagram photo sharing started initially with entertainment and social connectivity purposes in mind, its advantages have extended to education, marketing, business, health and many other areas (Huh et al. 2014; Kim et al. 2017). In the healthcare field, social media has generally played an important role in delivering explicit health messages. These include raising awareness, connecting health providers with patients, and helping patients connect with peers in order to obtain social and emotional support (Laranjo 2016).

Over time, users have moved gradually from posting only textual content about their experiences with chronic diseases, to posting photos that are mostly personal. However, there is a lack of research that investigates photo sharing on social networks related to chronic disease. Previous research refers to the role of visual content to deliver health messages and enhance health communication between patients in online health communities (O’Donnell \& Willoughby 2017; Rus \& Cameron 2016).

While there has been work done on online media for carers (Gleeson et al. 2017a), there is no research that investigates the influence of photo sharing on coping skills of patients who care for their children with chronic disease. Therefore, the aim of this study is to explore the effectiveness of using online photo sharing to support parents in coping with the conditions of their chronically ill children (particularly children with Type 1 diabetes). We use stress and coping theory as a lens to explore our work. This study is a stage of a larger project to explore chronic disease patients' engagement in photo sharing platforms.

In view of this aim, our research question is: How does online photo sharing support and influence the lived experiences of parents coping with caring for chronically ill children?

In this research we conduct semi-structured interviews and analysis of the Instagram postings of participants from online groups of parents of children with Type 1 Diabetes. We found that participants had a variety of objectives and coping strategies when using online photo sharing. While participants used photo sharing as a source of social support, they were also constrained by privacy concerns. This novel work, in a previously underexplored area, provides interesting findings for the design of photo sharing platforms.

\section{Literature Review}

\subsection{Photography and Online Photo Sharing Platforms}

Until recently, sharing of information using social media has been predominantly text based, with many illness groups represented in text-based online health communities (Fan et al. 2013; Fan et al. 2014; Fan and Lederman, 2018). However, the addition of photo sharing capabilities, which have altered the way people produce and share knowledge and provided opportunities for rich interactions online (Rose 2013).

The literature suggests that photo sharing is used to build relationships, for self-expression, for selfpresentation, and for information sharing (Beldad \& Hegner 2017; Huh et al. 2014). Users post photos to reflect their ideas (Eftekhar et al. 2014) and to present more precise forms of themselves (Beldad \& Hegner 2017). Users of online photos sharing platforms want to be linked to groups and feel a sense of belonging (Beldad \& Hegner 2017; Malik et al. 2015). and extend their social circle (Thelwall et al. 2015). This leads users to seek affection and attention in their circle by obtaining likes and comments on their photos thus validating their photo sharing efforts (Malik et al. 2015).

However, we know little about why or how photo sharing is used as a form of emotional support for specific health related communities. O'Donnell and Willoughby (2017) find that using photos to share health information is more effective than text especially when the target is young users through social networks. Photos discussing self-management or health information are particularly noted as having high user engagement through likes and comments (Paige et al. 2015).

\subsection{Social Support}

Photo sharing often takes place in online communities where many families of Type 1 diabetic individuals use social media to derive support from their peers (Ho et al. 2014). Support groups exist in the form of chatrooms, forums, mailing lists, blogs, groups on Facebook, hashtags, accounts on Twitter 
and Instagram. Social support from other parents is a strong motivation for participation (Gleeson et al. 2017b; Zhang et al. 2013). However, previous studies on the support provided by online diabetes communities focus on either local specialized portals controlled by professionals, or social media pages related to diabetes organizations (Merkel \& Wright 2012; Nordfeldt et al. 2013).

\subsection{Health Information and Personal Experience Sharing}

An increasing number of diabetes patients and their families prefer to search for health information themselves in online health communities rather than contacting their local clinics (Nordfeldt et al. 2013). This trend has led to an increasing number of moderated and non-moderated diabetes forums and social media groups, which are managed by non-government organizations (NGOs), governmental organisations, commercial agents, and individuals, with the information provided often difficult to understand and absorb (Dreyfus el al. 2011; Smith et al. 2012). Health diabetes information published in online forums and social media are either produced by professionals, created by users, or evidencebased (Fergie et al. 2016). Some studies suggest that patients and their families value the personal health experiences of peers more than the solid health information provided by health professionals (Zhang et al. 2013). In addition, adolescents with Type 1 diabetes and their parents engage in online diabetes programs and Facebook groups in order to increase their diabetes knowledge, peruse health information posted by health professionals and peers, and request disease-specific information and guidance (Ho et al. 2014; Petrovski et al. 2015).

Furthermore, some Diabetes foundations and organizations now encourage patients to engage in online posts (Hunt \& Koteyko 2015). For example, they use questions to invite users to contribute to discussions and share their experiences and opinions. This approach has significant implications for the way in which people deal with health information sharing. Patients have become more open and show more willingness to share personal health information and experiences and to identify themselves as diabetes patients (Giménez-Pérez et al. 2016; Hunt \& Koteyko 2015).

\subsection{Factors in Engagement}

The design of online forums has an impact on engagement. Users prefer user-friendly, accessible, and convenient websites to keep engaging with others (Fan et al. 2010; Ho et al. 2014; Lederman et al. 2014; Nicholas et al. 2012; Nordfeldt et al. 2013), while others prefer online communities that provide grouping features and one to one contact (Johnston et al. 2013). The tone of message content also impacts on engagement. A message with positive content is more likely to obtain a high number of likes and favourites than ones with negative content (Hunt \& Koteyko 2015). Moreover, image is a critical feature that influences the response to the message where positive image seems to be a motivational factor for user interaction and encourages users to participate in the discussion. Rus and Cameron (2016) indicate that messages with images have a greater possibility of obtaining more likes and shares than messages without images.

\subsection{Coping Theory}

In considering this literature we can see that it suggests a number of ways in which social media can assist parents in coping with their lived experience as carer for a sick child. Coping has been defined by the stress and coping theory as 'The cognitive and behavioural efforts made to master, tolerate, or reduce external and internal demands and conflicts among them" (Lazarus \& Folkman 1984). Coping skills can be distinguished by their focus, which include emotion-focused coping and problem-focused coping (Lazarus \& Folkman 1984). While problem-focused coping refers to identifying the cause of stress and dealing with stress through methods such as information seeking, problem solving, and obtaining instrumental social support, emotion-focused coping deals with stress by managing emotions as a result of stress such as writing diaries, seeking emotional support, avoidance, and suppression (Grey 2000; Lazarus \& Folkman 1984). For a chronic disease like Diabetes, it is important for the patient and the carer to engage in both forms of coping strategies (Grey 2000). However, since Type 1 Diabetes is a chronic disease and the source of stress is enduring, positive emotion-focused coping is more effective (Grey 2000).

\section{Research Design}

We use qualitative methods to collect and analyze interviews and Instagram posts. First, semistructured interviews were conducted over a four months period. Interviews are important to gather rich data from participants and to better understand the intentions behind specific behavior (Yin 2016). Following the interviews, 1,290 user's Instagram posts were collected over two months followed by an analysis of these posts for each participant. Participants' posts consist of their online photo sharing 
activities i.e. the photos, comments in accompanying posts and hashtags used. While there are several methods to deal with visual data, in this context, content analysis seems to be the ideal choice for many reasons. First, it deals with a large sample size (Pauwels 2012). Second, it is context focused to explore trending topics in the online platforms and makes it possible to deal with photo and text together (McMillan 2000).

Prior to commencing with the data collection, ethics was sought and granted by the Human Ethics Advisory Group (HEAG) of the Melbourne School of Engineering at the University of Melbourne (Ethics ID 1647308). Following ethics approval, the first author set out to recruit study participants- parents of at least one Type 1 diabetic child or adolescent. This choice is justified by the fact that parents are the main individuals responsible for their children' disease management and hence are more likely to have parental stress (Streisand et al. 2008). Parent needed to have spent at least three months already engaged in social media and on Instagram in particular. In addition, participants had to identify themselves on the Instagram profile as a parent of a Type 1 diabetic child and be actively posting photos on this platform. For recruitment, direct Instagram messaging was used allowing the sending of a short message as invitation to participate suggested through two hashtags \#t1dmom \#t1ddad. Only 10 mothers expressed their interest to participate. The mean age for the participants was 32 years and they were from four different countries. All participant data was de-identified using a pseudonym to refer to each participant (P1 to P10) as required by the ethics. Participant details are outlined in the table below:

\begin{tabular}{|c|c|c|c|c|c|c|}
\hline $\begin{array}{l}\text { Participant } \\
\text { Number }\end{array}$ & Gender & $\begin{array}{l}\text { Parent } \\
\text { Group }\end{array}$ & Age & $\begin{array}{l}\text { Child } \\
\text { Group }\end{array}$ & Age & Diagnosis \\
\hline P1 & Female & $41-45$ & & $10-13$ & & 2 years \\
\hline P2 & Female & $31-35$ & & $3-5$ & & 6 months \\
\hline $\mathbf{P}_{3}$ & Female & $26-30$ & & $6-9$ & & 2 years \\
\hline $\mathbf{P 4}$ & Female & $36-40$ & & $14-16$ & & 3 years \\
\hline $\mathbf{P}_{5}$ & Female & $36-40$ & & $6-9$ & & 2 years \\
\hline P6 & Female & $26-30$ & & $6-9$ & & 4 years \\
\hline $\mathbf{P}_{7}$ & Female & $21-25$ & & $1-2$ & & 6 months \\
\hline P8 & Female & $36-40$ & & $10-13$ & & 8 years \\
\hline P9 & Female & $31-36$ & & $6-9$ & & 4 years \\
\hline Pio & Female & $31-36$ & & $6-9$ & & 1 year \\
\hline
\end{tabular}

Table 1: Background information of participants

\subsection{Interviews}

Participants engaged in an individual 20 minutes online semi-structured interview on SKYPE to collect background information, to understand their intentions in using Instagram, to share their experience as parents of children with Type 1 diabetes and to obtain rich information about their positive and/or negative experiences of using Instagram. Interviews aimed to examine whether parents could obtain the social support they needed, and to what extent Instagram enabled them to cope with their children's conditions as a result of online engagement. The questions were open ended allowing participants to reflect on their journey through photo sharing. Table 2 shows a sample of the interview questions. Each interview is analyzed into corresponding codes, categories, and themes (Miles \& Huberman 1994). Themes emerged from the interviews and the posts' text were classified based on the application of coping theory to identify any coping skills.

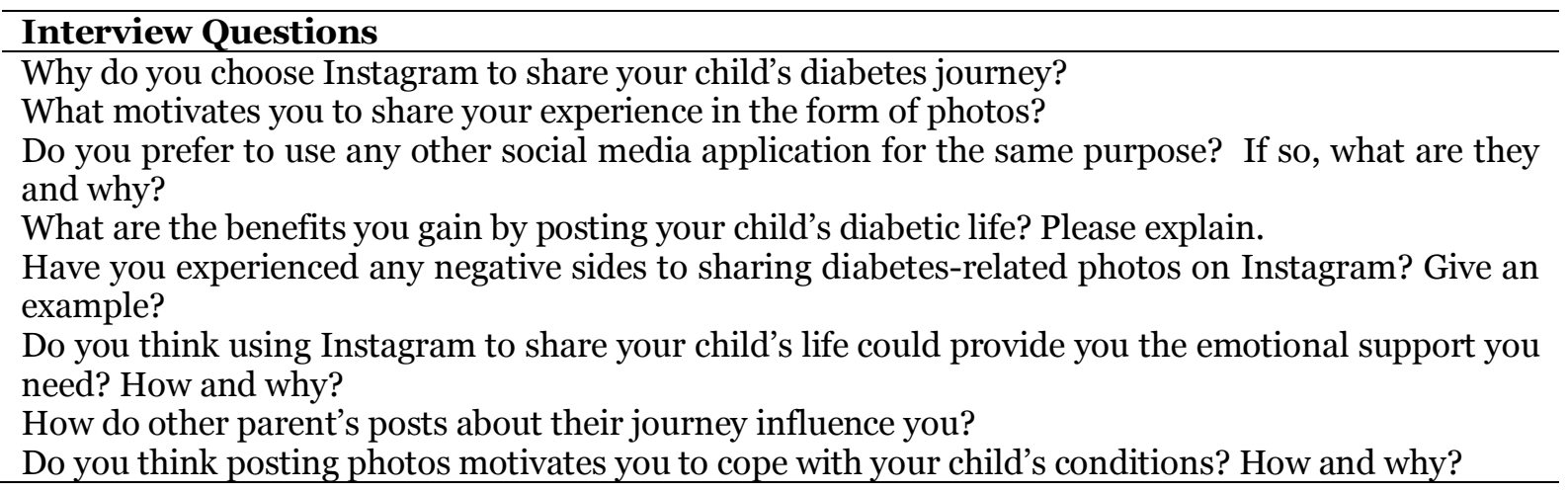

Table 2: A sample of the interview questions. 


\subsection{Instagram Account Data}

The second source of data was Instagram posts shared with others. This included each participant's posts of the following; photos, captions, comments, hashtags and likes. We found 1,290 posts that were related to diabetes across the 10 accounts. The range of diabetes-related posts was between 12 to 579 for each account (see Table 3 for a statistical analysis of the topics discussed in the participants accounts).

This content was extracted from each participant's account on Instagram. To reduce the amount of the data, only diabetes-related posts were included. To ensure including all of these different types of data, quantitative and qualitative analysis was conducted.

Posts and photos collected from Instagram were analyzed as follows:

- First, we performed a discrete statistical analysis to obtain an overview of interaction in the account including number of followers, the commenting mean, and the 'like(s)' mean.

- Second, we labelled each photo by manually interpreting its content. Then categories are created to reflect the photos' content (Table 3).

- Third, we coded the textual content of each post that including captions and comments.

- Last, the themes that emerged from an analysis of the posts' content were analyzed by comparing them with the coding themes that emerged from the interviews. The last step was important from a validity perspective to ensure that both instruments lead to the same findings and interpretations.

The initial statistical analysis showed differences in the most discussed topics for each account based on their objectives. In addition, the number of followers and the purpose of the accounts highly affected the way people commented and liked any post. The most significant discussed topics were related to raising awareness and sharing positive everyday activities related to parent's caring for their children.

\begin{tabular}{|c|c|c|c|c|c|c|c|c|c|c|c|c|c|c|c|}
\hline \multirow{2}{*}{ 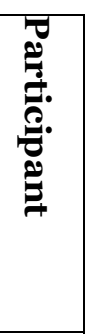 } & \multirow[b]{2}{*}{ 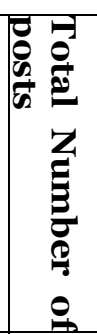 } & \multirow[b]{2}{*}{ 量 } & \multirow{2}{*}{ 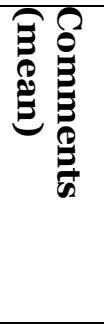 } & \multirow{2}{*}{ 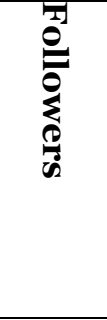 } & \multicolumn{11}{|c|}{ Posts Topics (number of posts) } \\
\hline & & & & & 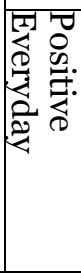 & 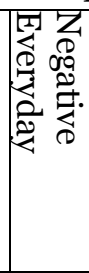 & 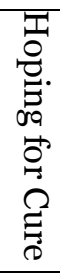 &  & 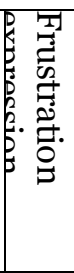 & 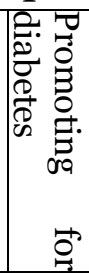 &  & 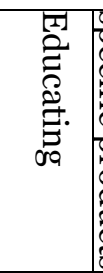 & 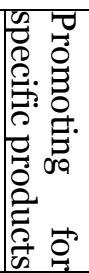 &  & 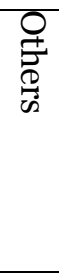 \\
\hline P1 & \begin{tabular}{|l|}
89 \\
\end{tabular} & 14.73 & 1.86 & 184 & 21 & 9 & 11 & 0 & 13 & 15 & 7 & 8 & 0 & 5 & 0 \\
\hline P2 & 12 & 27.09 & 2.16 & 236 & 3 & 4 & 1 & 0 & 0 & 0 & o & 2 & 2 & 0 & 0 \\
\hline $\mathrm{P}_{3}$ & \begin{tabular}{|l|}
107 \\
\end{tabular} & 15.76 & 0.75 & 266 & 27 & 13 & 9 & 0 & 11 & 17 & 9 & 5 & 13 & 3 & 0 \\
\hline $\mathrm{P} 4$ & 91 & 17.45 & 1.76 & 760 & 21 & 14 & 16 & $\mathrm{O}$ & 9 & 2 & 18 & 2 & $\mathrm{O}$ & 9 & 0 \\
\hline $\mathrm{P}_{5}$ & 19 & 23.86 & 2.6 & 1081 & 2 & 7 & 1 & $\mathrm{O}$ & 8 & $\mathrm{O}$ & 1 & 0 & 0 & 0 & $\mathrm{O}$ \\
\hline P6 & 28 & 306.50 & 17.67 & 13844 & 4 & o & 0 & 0 & 0 & 7 & 2 & 8 & 7 & 0 & 0 \\
\hline $\mathrm{P}_{7}$ & 141 & 17.71 & 5.48 & 1529 & 0 & 1 & 8 & 34 & 3 & 0 & 43 & 49 & 3 & 0 & 0 \\
\hline P8 & 579 & 33.58 & 6.37 & 2504 & 11 & 5 & 12 & 146 & 0 & 15 & 141 & 203 & 12 & 16 & 18 \\
\hline P9 & 187 & 22.19 & 2.89 & 2045 & 2 & 9 & 18 & 38 & 8 & 3 & 37 & 64 & O & 0 & 8 \\
\hline P10 & 37 & 17.88 & 2.76 & 82 & 7 & 3 & 1 & 3 & 0 & 0 & 11 & 8 & 4 & 0 & 0 \\
\hline
\end{tabular}

Table 3: Initial statistical analysis for the topics discussed in the participants accounts.

\section{Findings}

This section describes three themes that emerged from the qualitative data analysis (i.e. interpretations of the interview data) in combination with the interpretation of the topics that emerged from data analysis of the content of Instagram posts.

\subsection{Coping Strategies Through Photo Sharing Platforms}

Parents in this study indicated that their adoption of photo sharing helped them to develop many coping strategies to reduce parental stress related to their children's conditions. Many of these strategies were enabled by social media where most of the coping strategies are positive emotion-focused: i) having gratitude for technological, human and spiritual supports; ii) visualizing their children's positive activities, and iii) emotional social support. In addition, problem-focused coping strategies occur in limited activities online including: i) engaging and raising awareness of diabetes campaigns and ii) educating other parents who have children with Type 1 diabetics. 


\subsubsection{Positive emotion-focused coping}

- Sharing a visual dairy and experience through photos

Participants appreciated the experience of sharing their life through photos. In fact, there was a conflict between the way they talked about the positivity of their experience in the interviews and the fact that more than half of the shared photos in their accounts did not get high numbers of comments or likes. However, participants explained the real relief they felt when going through old photo posts. They commented on the progress they've made in their journey which gave them a motive to provide more support for their children and empower them to cope with their situation:

"Everything in my whole life is online including my son, what we're going through and challenges since we were at the hospital, I like [it] when I go back to see where we were and what we are doing now" (P1, Q1) (key: P1=participant 1, Quote 1).

In addition, sharing diabetes experiences has many forms such as: sharing how parents suspected the disease to unfold and symptoms experienced, pictures since the first day of diagnoses and feelings about that, and how parents dealt with the highs and lows associated their children's every-day diabetes challenges.

"This was the day when everything changed. My baby girl was diagnosed with Type 1 Diabetes and our world was turned upside down... It was her teacher that noticed she was losing weight, drinking a lot of water \& just wasn't acting like herself... I worried so much " $\left(\mathrm{P}_{4}, \mathrm{Q} 2\right)$.

- Expressing feelings of gratefulness for sources of support

Another emotional coping strategy used by the participants was through posting to other parents how they were grateful to the source(s) of support in their lives. Sources of support specifically mentioned included spiritual, diabetes-related technologies, partners, and other family members.

"I am NOT thankful for this chronic disease in our family life that hit us like a bus 8 months ago. But I am becoming grateful for the strength God has given us to handle what we were given" ( $\left.\mathrm{P}_{5}, \mathrm{Q} 3\right)$.

Moreover, advanced diabetes control devices made dealing with diabetes easier, especially for parents. Although they were not available for all, parents who could benefit from them were thankful for their existence and encouraged other parents to try using these devices.

In addition, participants always referred to the support of their partners and other siblings in helping to care for their diabetic children and how this support could reduce parental stress.

"My oldest treated her sister, low [hypoglycaemia] and laid with her to make sure she didn't miss an alarm." (P1, Q4)

- Picturing diabetic children's positive activities

Sharing photos of the regular life of diabetic children was clear accounts for a range of between $2 \%$ and $29 \%$ in total posts for individual accounts. Participants' posts were varied in the way they showed the personal life of their children related to diabetes. Across all posts, parents always tried to show the positive side of everyday activities, and that diabetes would not stop their children participating in regular activities. An analysis of Instagram posts aimed to identify sentiments of positive and negative activity as shown from the evidence below:

Positive activity: "My sweet girl is off to D-camp! First away camp ever! so proud of her" (P1, Q5)

Negative activity: "I despise changing Dexcom sensors... No amount of breathing or counting will ever make that pit in my stomach go away..." (P4, Q6)

- Direct and indirect emotional social support

Through the interviews and Instagram posts, participants referred to different forms of social support through Instagram. Although the majority of the participants sought emotional support from other parents on Instagram, two of them declared that they use Instagram to provide support for others, as they do not need it themselves as they have moved on in the coping journey.

Participants who sought emotional and social support claim they use hashtags and search features in Instagram to reach out to other parents and Type 1 diabetes patients. They obtain emotional support either through positive comments and discussions on their posts or through private conversation using the Instagram's Direct Message feature. 
"To all my T1 mama bears! You are awesome! Thank you for being my support! I love you and you should love yourselves more!" (P4, Q7)

The majority of the participants referred to another form of emotional and social support where they introduce themselves to other parents on Instagram and then create new support groups through other social media applications such as Facebook and WhatsApp that provide more private environments and an easier way to discuss their children's issues and how to deal with them

"Instagram helped me to get contacted with other moms and specialists who also have diabetes through WhatsApp" (P6, Q8)

\subsubsection{Problem-focused coping}

- Seeking health information and personal experience

Participants show the need to obtain more information even though they may trust information from health providers. They look for more information and personal experience on Instagram to obtain feedback from other parents about specific conditions or supplies.

"I connect with health professionals' profiles and other moms. Sometimes to check information for me and my kid and to know about new technologies like pumps that we're not using yet whether it is comfortable or not." ( $\left.\mathrm{P}_{4}, \mathrm{Q} 9\right)$

- Engaging in diabetes campaigns and raising awareness

This strategy was considered one of the favourite themes in posts of all participating parents. They believed they could make a difference by raising awareness about the disease's symptoms and its complications. The main target for the majority of the participants was family and friends as they discussed diabetes in their accounts to show their family and their children's friends what the daily diabetes experience looks like.

"My friends and family are interested to know [more] about diabetes... Several of her [my child's] friends follow me. Now I just want them to understand" ( $\left.\mathrm{P}_{3}, \mathrm{Q} 10\right)$

Raising awareness posts ranged between posting on diabetes health information, photos of parents with their children engaging in diabetes campaigns, and posts to others encouraging donations for diabetes organisations, or using trending hashtags about diabetes.

- Educating other parents about Type 1 diabetes

The last coping strategy used by participating parents is posts to educate other parents about Type 1 diabetic children. The most discussed topics are feedback about the latest technology related to diabetes, and nutrition information and how to count carbohydrates and deal with highs and lows based on their experiences.

"My purpose is to educate other moms in order to get better management of diabetes." (P9, Q11)

\subsection{Photos as a Preference}

The majority of the participants emphasized that photos have a greater impact than text alone. In particular photos sharing individual journeys of hardship and angst parents experienced as a result of caring for their children strengthened social ties in the network.

"I want them to see it through my eyes and sometimes words don't always give the justice that you see on pictures..." (P1, Q12)

Photos were referred to as a 'shortcut' to reading a whole article - one could write a few words with a photo that describes everything. The importance of photos was not limited to only parents of diabetic children, but also Type 1 diabetic children, as photos were considered a language that was understandable by all people.

On the other hand, participating parents preferred to use Instagram due to its accessibility to different age groups, its user-friendly interface, ease-of-use, and the availability of hashtags and search features that helped to reach other parents of Type 1 diabetics accounts.

"Instagram is pretty user friendly and I am most comfortable to use it. It allows me to search for people who like me using hashtags about diabetes, type one diabetes, insulin dependent and then we can start conversation" ( $\left.\mathrm{P}_{4}, \mathrm{Q} 13\right)$ 
Statistical analysis of posts confirmed the affective use of photos to deliver messages and to attract other users to read, comment, and like the posts.

\subsection{Benefit of Personal Photo Sharing}

Many participants referred specifically to the benefit of personal photos of real people as a way to get their message across and provide validity to the information they were sharing:

"I saw many Kuwaiti moms accounts who talk about diabetes but do not share their children photo, so I could not see the impact as when the child speaks. You can see his own life what looks like." (P10, Q14)

"My family and my mother could not understand what we are going through. However, photos now could explain everything, and they understand the situation and how is the life with diabetes" (P10, Q15)

\section{Discussion}

We see in this study that participants used Instagram as a coping strategy in different ways that include sharing personal experience with other parents, picturing positive everyday activities, engaging in diabetes campaigns online, raising awareness, and encouraging others to donate for diabetes research organisations. Some parents describe the purpose of their accounts as purely educational while others refer to an opportunity to share daily life moments with diabetes with close family members and peers (see Q5, Q15). The third group posts photos to specifically seek social support in their journey on adapting to caring for children with Type 1 diabetes. This behaviour is consistent with the literature (Rus and Cameron 2016) which suggest that posts with personal experiences or the seeking of emotional support are likely to obtain higher engagement. Personal photos can be used to describe participants' own and their children's experiences which is important to describe the situation to family and friends, raise awareness about the disease, and raise donations for diabetes research (see Q10). The increase in the willingness to share more personal health information online is supported by Hunt \& Koteyko (2015). Moreover, participating parents refer to sharing photos that explain their experiences with the disease as a 'relief', although some of the photos did not attract the expected number of likes or comments (see Q1).

Participants posted concerns about privacy and trustworthiness issues. Through the content analysis of postings on participants' accounts, there were significant behaviours identified related to problematic privacy practices, particularly where photos were accompanied by text. The majority of the participants openly shared their names and children's names, their children's health situation and regular check results, as well as complications related to their children's chronic disease. Although it has become more common behaviour on social media recently to share openly (Malik et al. 2015), the effect of this behaviour is not fully known.

Participants refer to the ease with which they can deal with photos technically and emotionally (see Q18). These findings suggest two forms of emotional social support through Instagram, i.e. direct and indirect social support. Direct emotional social support is attained through likes, positive comments, expressions of empathy, well wishes, and hope for cures in discussion, which are similar to what Zhang et al. (2013) found in their study. Indirect social support through Instagram is identified in this study as participating parent's use of Instagram hashtags and search features allowing individuals to find other parents and invite them to join private online diabetes discussion and collaboration groups through Facebook and WhatsApp. These groups provide more private and specialised environments for parents of Type 1 diabetics that Instagram does not offer.

Significantly, the value of photo sharing relies on the capabilities of the social media application. Findings indicate the features of the application that encourage or discourage people from using it. While there are multiple online photo sharing platforms, not all of them are favoured by the parents in the sample. The ability to provide temporal sequences of photos creates a story line for parents who prefer to review how they deal with the disease over time. The ability to capture and post photos with the least expected technical issues is another key factor. This factor is consistent with the literature where Ho et al. (2014) and Nicholas et al. (2012) mention that users prefer user-friendly and convenient online platforms. The last factor is the value of searching through hashtags that allows patients with any disease and their families to meet and find peers all over the world. In addition, participants all agree that visual posts including photos and short videos have more power in delivering their messages than other tools that include text and longer videos. Rus and Cameron (2016) refer to this point in their study 
indicating that posts with photos attract more user interaction and engagement. They see photos as presenting much more information than text.

Participants suggest that using online photo sharing helped them to create new relationships with other parents around the world. In addition, initial photo sharing can create more trust between participants where they go on to invite parents to closed groups through WhatsApp and Facebook. Fan and Lederman (2018) indicate that trust in online health communities is influenced by the interaction level between the users. Our study suggested that parents look for peers through their online photo sharing activities where they build more trust to engage in private communication. Due to privacy concerns, this finding shows a new style of communication on online communities where there is no moderator to control the platform.

Photo sharing provides opportunity for relationship building that could not be easily achieved otherwise. Participants in this study recognize themselves in profile information as parents with Type 1 diabetic child which helps to create possible new ties with other parents who visited their profiles as well as using hashtags to further increase access to others in a shared community of interest. This finding is supported by Beldad \& Hegner (2017) where they find users normally share photos online to create new social ties and relationships.

Most significant to the role of coping theory in the context under examination is the claim that personal real photos provide a more valid or more substantiated form of evidence than text in the online context $(\mathrm{Q} 14,15)$. Findings indicate different emotion-focused coping skills compared to limited problemfocused coping through photos which could be linked to the nature of Type 1 Diabetes as a chronic disease. With regard to coping theory, photos were seen to substantiate the truth of the personal experience more than text and thus generate easier ways to cope with emotions (Q1-Q8). While tentative in this limited context, this is a significant and important finding suggesting the greater possible power of photo sharing in positive emotions-focused coping than text based media.

\section{Conclusion}

This study explores the support provided to carers of children with Type 1 diabetes by online photo sharing platforms. This study finds that photos create a storyline for their experience as well as an opportunity to express their positive and negative feelings about their experience, to share strategies and personal experiences, to discuss sources of support, to create positivity and to build awareness of both the condition and fundraising opportunities. The paper also explores the idea of coping through digital photo sharing. Our contribution is to consider the idea of coping with stress in this specific context of photo-sharing in a health community. Our tentative finding is that such platforms can be designed to facilitate engagement with other patients and the adoption of coping skills related to health issues. This finding is worthy of further exploration.

\section{Limitations}

This paper has limitations due to the small sample size and its focus on only a group of parents with a specific chronic disease. The study is limited to the case of parents of children with Type 1 diabetes in online photo sharing and may not be applicable in other situations due to the small sample size and using the use of qualitative research tools.

Parents with more than one child with Type 1 diabetes or family history with diabetes are excluded from the study. The findings are based on a single photo-sharing platform and did not discuss user engagement in other photo based social media such as Facebook and Twitter. This study uses coping and stress theory to explore the effectiveness of using online photo sharing in health. However, it excludes the influence of multiple social factors such as socio-economic status. Moreover, this study cannot be generalized due to the previous reasons in addition to its dependence on qualitative data.

\section{References}

Beldad, A. D., and Hegner, S. M. 2017. "More Photos from Me to Thee: Factors Influencing the Intention to Continue Sharing Personal Photos on an Online Social Networking (OSN) Site among Young Adults in the Netherlands," International Journal of Human-Computer Interaction (33:5), Taylor \& Francis, pp. 410-422. (https://doi.org/10.1080/10447318.2016.1254890).

Dreyfus, S., Lederman, R. M., Smith, S. P., and Monagle, P. T. 2011. "Customising pathology report design for patient use". eJournal of Health Informatics, 6 (2). 
Eftekhar, A., Fullwood, C., and Morris, N. 2014. "Capturing Personality from Facebook Photos and Photo-Related Activities: How Much Exposure Do You Need?, Computers in Human Behavior (37), Elsevier Ltd, pp. 162-170. (https://doi.org/10.1016/j.chb.2014.04.048).

Fan, H., Lederman, R., Smith, S., and Chang, S., 2010. "Why People Trust in Online Health Communities: An Integrated Approach", 21st Australasian Conference on Information Systems, December 1-3.

Fan, H., Lederman, R., Smith, S., and Chang, S., 2013. "How Online Health Forum Users Assess UserGenerated Content: Mixed-Method Research”, European Conference in Information Systems.

Fan, H., Lederman, R., Smith, S., and Chang, S., 2014. "How Trust Is Formed in Online Health Communities: A Process Perspective", Communications of the Association for Information Systems: Vol. 34, Article 28.

Fan, H., and Lederman, R. 2018. "Online Health Communities: How Do Community Members Build the Trust Required to Adopt Information and Form Close Relationships?," European Journal of Information Systems (27:1), Taylor \& $\quad$ Francis, pp. 62-89. (https://doi.org/10.1080/0960085X.2017.1390187).

Fergie, G., Hilton, S., and Hunt, K. 2016. "Young Adults' Experiences of Seeking Online Information about Diabetes and Mental Health in the Age of Social Media," Health Expectations (19:6), pp. 1324-1335. (https://doi.org/10.1111/hex.12430).

Giménez-Pérez, G., Recasens, A., Simó, O., Aguas, T., Suárez, A., Vila, M., and Castells, I. 2016. "Use of Communication Technologies by People with Type 1 Diabetes in the Social Networking Era. A Chance for Improvement," Primary Care Diabetes (10:2), Primary Care Diabetes Europe, pp. 121-128.

Gleeson, J., Koval, P., Eleftheriadis, D., Lederman, R., Herrman, H., Bendall, S., Cotton, SM., \& AlvarezJimenez, M. 2017. "Moderated online social therapy for carers of young people recovering from first-episode psychosis: study protocol for a randomised controlled trial”, TRIALS, vol. 18.

Gleeson, J., Lederman, R., Koval, P., Wadley, G., Bendall, S., Cotton, S., Herman, H., Crisp, K. \& AlvarezJimenez, M. 2017. Moderated Online Social Therapy: A Model for Reducing Stress in Carers of Young People Diagnosed with Mental Health Disorders. Frontiers in Psychology, 8, 485-497.

Grey, M. 2000. "Lifestyle and Behavior: Coping and Diabetes," Diabetes Spectrum (13:3), p. 167.

Ho, Y.-X., O'Connor, B. H., and Mulvaney, S. A. 2014. "Features of Online Health Communities for Adolescents with Type 1 Diabetes," Western Journal of Nursing Research (36:9), pp. 1183-1198. (https://doi.org/10.1177/0193945913520414).

Huh, J., Liu, L. S., Neogi, T., Inkpen, K., and Pratt, W. 2014. "Health Vlogs as Social Support for Chronic Illness Management," ACM Transactions on Computer-Human Interaction (21:4), pp. 1-31. (https://doi.org/10.1145/2630067).

Hunt, D., and Koteyko, N. 2015. "What Was Your Blood Sugar Reading This Morning?' Representing Diabetes Self-Management on Facebook," Discourse \& Society (26:4), pp. 445-463. (https://doi.org/10.1177/0957926515576631).

Instagram. 2018. "Press News.” (https://www.instagram.com/press/?hl=en).

Johnston, A. C., Worrell, J. L., Di Gangi, P. M., and Wasko, M. 2013. "Online Health Communities," Information Technology \& People (26:2), pp. 213-235. (https://doi.org/10.1108/ITP-02-20130040).

Kim, D. H., Seely, N. K., and Jung, J. H. 2017. "Do You Prefer, Pinterest or Instagram? The Role of Image-Sharing SNSs and Self-Monitoring in Enhancing Ad Effectiveness," Computers in Human Behavior (70), Elsevier Ltd, pp. 535-543. (https://doi.org/10.1016/j.chb.2017.01.022).

Laranjo, L. 2016. "Social Media and Health Behavior Change," Participatory Health through Social Media, Elsevier Inc. (https://doi.org/10.1016/B978-0-12-809269-9.00006-2).

Lazarus, R. \& Folkman, S. 1984. Stress, Appraisal, and Coping, New York: Springer Pub. Co. (https://doi.org/10.1037//o033-2909.116.2.340).

Lederman, R. M., Wadley, G. R., Gleeson, J., Bendall, S., and Alvarez, M. 2014. "Moderated online social therapy: Designing and evaluating technology for mental health". ACM Transactions on Computer-Human Interaction, 21(1). 
Malik, A., Dhir, A., and Nieminen, M. 2015. "Uses and Gratifications of Digital Photo Sharing on Facebook," Telematics and Informatics (33:1), pp. 129-138. (https://doi.org/10.1016/j.tele.2015.06.009).

McMillan, S.J. 2000. "The microscope and the moving target: The challenge of applying content analysis to the World Wide Web," Journalism \& Mass Communication Quarterly, 77(1), pp.8098.

Merkel, R. M., and Wright, T. 2012. "Parental Self-Efficacy and Online Support among Parents of Children Diagnosed with Type 1 Diabetes Mellitus," Pediatr Nurs (38:6), p. 303-8; quiz 309. (https://doi.org/10.1016/j.pedhc.2015.08.002).

Miles, M. B., and Huberman A. M. 1994. An Expanded Sourcebook: Qualitative Data Analysis (2nd $E d$.$) . Thousand Oaks, CA, Sage Publications.$

Nicholas, D. B., Fellner, K. D., Frank, M., Small, M., Hetherington, R., Slater, R., and Daneman, D. 2012. "Evaluation of an Online Education and Support Intervention for Adolescents with Diabetes.," Social Work in Health Care (51:9), pp. 815-27. (https://doi.org/10.1080/00981389.2012.699507).

Nordfeldt, S., Ängarne-Lindberg, T., Nordwall, M., and Krevers, B. 2013. "Parents of Adolescents with Type 1 Diabetes - Their Views on Information and Communication Needs and Internet Use. A Qualitative Study," PLOS ONE (8:4). (https://doi.org/10.1371/journal.pone.0062096).

O'Donnell, N. H., and Willoughby, J. F. 2017. "Photo-Sharing Social Media for eHealth: Analysing Perceived Message Effectiveness of Sexual Health Information on Instagram," Journal of Visual Communication in Medicine (40:4), Informa UK Limited, trading as Taylor \& Francis Group, pp. 149-159. (https://doi.org/10.1080/17453054.2017.1384995).

Paige, S. R., Stellefson, M., Chaney, B. H., and Alber, J. M. 2015. "Pinterest as a Resource for Health Information on Chronic Obstructive Pulmonary Disease (COPD): A Social Media Content Analysis," American Journal of Health Education (46:4), pp. 241-251. (https://doi.org/10.1080/19325037.2015.1044586).

Pauwels, L. .2012. "Contemplating the State of Visual Research: An Assessment of Obstacles and Opportunities". Advances in Visual Methodology, 3-24. (https://doi.org/10.4135/9781446250921)

Petrovski, G., Zivkovic, M., and Stratrova, S. S. 2015. "Social Media and Diabetes: Can Facebook and Skype Improve Glucose Control in Patients with Type 1 Diabetes on Pump Therapy? One-Year Experience," Diabetes Care (38:4), pp. e51-e52. (https://doi.org/10.2337/dc14-2487).

Rose, G., 2013. Visual methodologies: An introduction to researching with visual materials. sage

Rus, H. M., and Cameron, L. D. 2016. "Health Communication in Social Media: Message Features Predicting User Engagement on Diabetes-Related Facebook Pages," Annals of Behavioral Medicine (50:5), Annals of Behavioral Medicine, pp. 678-689. (https://doi.org/10.1007/s1216o016-9793-9).

Smith, S. P., Lederman, R. M., Monagle, P. T., Alzougool, B. M., Naish, L., and Dreyfus, S. 2012. Individually tailored client focused reports for ubiquitous devices: An experimental analysis. In the 23rd Australasian Conference on Information Systems ACIS.

Streisand, R., Mackey, E. R., Elliot, B. M., Mednick, L., Slaughter, I. M., Turek, J., and Austin, A. 2008. "Parental Anxiety and Depression Associated with Caring for a Child Newly Diagnosed with Type 1 Diabetes: Opportunities for Education and Counselling," Patient Education and Counselling (73:2), pp. 333-338. (https://doi.org/10.1016/j.pec.2008.06.014).

Thelwall, M., Stuart, E., Goriunova, O., Vis, F., Burns, A., Faulkner, S., and D'Orazio, F. 2015. "Chatting Through Pictures? A Classification of Images Tweeted in One Week in the UK and USA". Journal Of The Association For Information Science And Technology, 67(11), pp. 2575-2586.

Yin, R. K. 2016. Qualitative Research from Start to Finish. New York. Guilford Press.

Zhang, Y., He, D., and Sang, Y. 2013. "Facebook as a Platform for Health Information and Communication: A Case Study of a Diabetes Group," Journal of Medical Systems (37:3). (https://doi.org/10.1007/s10916-013-9942-7). 